Auszahlungen der Zuweisungen aus dem Gesundheitsfonds in monatlich gleichen Teilbeträgen erfolgen.

Die Ortskrankenkassen verzeichnen einen Überschuss von rd. 627 Mio. Euro, die Ersatzkassen von 518 Mio. Euro, die Betriebskrankenkassen von 113 Mio. Euro, die Innungskrankenkassen von 121 Mio. Euro und die Knappschaft Bahn-See von 78 Mio. Euro. Nach Berechnungen des sog. GKVSchätzerkreises wird der Gesundheitsfonds Ende 2011 über eine Liquiditätsreserve von etwa 6,9 Mrd. Euro verfügen.

\section{MMW Kommentar}

Angesichts des regional bereits realen und in den nächsten fünf Jahren in dramatischer Form zu erwartenden Hausärztemangels stellt sich die Frage, warum nicht Teile dieses Überschusses gezielt für strukturelle Maßnahmen zur Verfügung gestellt werden, die in der Lage sind, diese Entwicklung zu brem- sen. Nichts davon ist jedenfalls im ab 1.1.2012 in Kraft tretenden sog. Versorgungsgesetz zu lesen. §87b Absatz 3 SGB V sieht vor, dass wenn der Landesausschuss der Ärzte und Krankenkassen regional eine Unterversorgung feststellt, für Ärzte der betroffenen Arztgruppe im Verteilungsmaßstab keine Maßnahmen zur Fallzahlbegrenzung oder-minderung bei der Behandlung von Patienten des betreffenden Planungsbereiches Anwendung finden dürfen. Die Geldmittel, die zu einer solchen Fördermaßnahme notwendig sind, werden aber nicht zur Verfügung gestellt. Im Gegenteil: §87a Absatz 4 SGB V sieht vielmehr vor, dass die Anpassung der vertragsärztlichen $\mathrm{Ho}^{-}$ norare auf Basis der vorliegenden aktuellen Daten über die Menge der abgerechneten Leistungen, die vier Kalendervierteljahre umfassen, jeweils nach sachlich-rechnerischer Richtigstellung und Anwendung honorarwirksamer Begrenzungsregelungen, für die
Versicherten der jeweiligen Krankenkasse mit Wohnort im Bezirk der Kassenärztlichen Vereinigung erfolgt.

Dies bedeutet, dass die Leistungen, die im zurückliegenden Jahr abgestaffelt vergütet wurden, auch weiterhin abgestaffelt bezahlt werden und aus diesem Volumen auch noch die o.g. Förderung abgezogen wird. Die KVen können diesbezüglich zwar einen Strukturfonds bilden, der 0,1\% der Gesamtvergütung beinhaltet. In diesem Fall müssen die Kassen zusätzlich einen Beitrag in gleicher Höhe leisten. Dies ergibt dann an „frischem Geld" allerdings nur eine lächerlich geringe Summe, die nie und nimmer dazu geeignet ist, die strukturellen Probleme in der ambulanten Versorgung zu beseitigen. Die Zeche zahlen die Vertragsärztinnen und -ärzte somit selbst. Wie man so Studienabgänger gewinnen will, die bereit sind, sich als Hausärzte zu engagieren, bleibt zunächst das Geheimnis unserer Gesundheitspolitiker!

\title{
Ab 1.7.2011 entscheidet der Patient über die Berichtspflicht
}

- Nach einem aktuellen Beschluss des Bewertungsausschusses setzt die Übermittlung der Behandlungsdaten und Befunde eines Patienten gemäß §73 Abs. 1b SGB V künftig voraus, dass hierzu eine schriftliche Einwilligung des Versicherten vorliegt, die sogar widerrufen werden kann. Gibt der Versicherte künftig auf Nachfrage keinen Hausarzt an bzw. ist eine schriftliche Einwilligung zur Information des Hausarztes nicht erteilt, sind die EBM-Gebührenordnungspositionen, bei denen bisher eine Berichtspflicht bestanden hat, auch ohne schriftliche Mitteilung an den Hausarzt berechnungsfähig.

Hintergrund für die Änderung des EBM ist ein Schreiben des Bundesbeauftragten für den Datenschutz und die Informationsfreiheit, in dem ausgeführt wird, dass mit der bisherigen Formulierung der Allgemeinen Bestimmung 2.1.4 des EBM die geltende Rechtslage nicht eindeutig wiedergege- ben wird. Zur Wahrung des Rechts auf informationelle Selbstbestimmung der Versicherten sei durch Beschluss des Bewertungsausschusses zu verdeutlichen, dass die ihn behandelnden Ärzte nach $\$ 73$ Abs.1b Satz 2 SGB V nur mit schriftlicher Einwilligung des Versicherten, die widerrufen werden kann, die in Satz 1 der Norm genannten Daten übermitteln dürfen. Hierbei soll insbesondere die datenschutzrechtliche Terminologie ("schriftliche Einwilligung" statt "Genehmigung") übernommen werden.

\section{MMW Kommentar}

Die Datenschützer scheinen sich auf ihre Fahnen geschrieben zu haben, die den Patienten vor Doppel- und Fehluntersuchungen schützende Lotsenfunktion des Hausarztes, wo immer es geht, zu unterwandern. Aus Datenschutzgründen wurden zunächst die Hausarztzentrierungsverträge torpe- diert und nun auch noch die hausärztliche Tätigkeit außerhalb dieser Selektivverträge nach §73b SGB V. Was sollte den nach dem 1.7.2011 einen fachärztlich tätigen Kollegen noch motivieren, sich die Arbeit zu machen, der 70-jährigen Oma, die vom Hausarzt zu einer notwendigen gebietsärztlichen Untersuchung überwiesen wurde, eine schriftliche Einwilligungserklärung abzuverlangen, die ihn dann auch noch zwingt, einen Arztbrief zu schreiben.

Pikant ist dabei, dass dieser Beschluss des Bewertungsausschusses keinerlei Angaben zur Bewertungsbereinigung der betreffenden Leistungen macht. Diese enthalten nämlich den Arztbrief kalkulatorisch. Wird dieser nicht mehr erbracht, steigt das Honorar für die Leistung durch Wegfall der Gegenleistung. 